\title{
On Some Pursuit and Evasion Differential Game Problems for an Infinite Number of First-Order Differential Equations
}

\author{
Abbas Badakaya Ja' afaru$^{1}$ and Gafurjan Ibragimov ${ }^{1,2}$ \\ ${ }^{1}$ Department of Mathematics, Universiti Putra Malaysia, Serdang, 43400 Selangor, Malaysia \\ 2 Department of Mathematics and Institute for Mathematical Research, Universiti Putra Malaysia, \\ Serdang, 43400 Selangor, Malaysia \\ Correspondence should be addressed to Abbas Badakaya Ja'afaru, badakaya@yahoo.com
}

Received 25 January 2012; Accepted 6 May 2012

Academic Editor: Debasish Roy

Copyright (c) 2012 A. B. Ja'afaru and G. Ibragimov. This is an open access article distributed under the Creative Commons Attribution License, which permits unrestricted use, distribution, and reproduction in any medium, provided the original work is properly cited.

\begin{abstract}
We study pursuit and evasion differential game problems described by infinite number of firstorder differential equations with function coefficients in Hilbert space $l_{2}$. Problems involving integral, geometric, and mix constraints to the control functions of the players are considered. In each case, we give sufficient conditions for completion of pursuit and for which evasion is possible. Consequently, strategy of the pursuer and control function of the evader are constructed in an explicit form for every problem considered.
\end{abstract}

\section{Introduction}

The books by Friedman [1], Isaacs [2], Krasovskiř and Subbotin [3], Lewin [4], Petrosyan [5], and Pontryagin [6] among others are fundamental to the study of differential games.

Many works are devoted to differential game problems described by both ordinary differential equations in $R^{n}$ and partial differential equations. In particular, pursuit and evasion differential game problems involving distributed parameter systems are of increasing interest (see, e.g., [7-14]).

Satimov and Tukhtasinov [10,11] studied pursuit and evasion problems described by the parabolic equation

$$
z_{t}-A z=-u+v,\left.\quad z\right|_{t=0}=z_{0}(x),\left.\quad z\right|_{S_{T}}=0,
$$

where $z=z(t, x)$ is unknown function; $x=\left(x_{1}, x_{2}, \ldots, x_{n}\right) \in \Omega \subset R^{n}, n \geq 1$ is parameter in a bounded domain $\Omega ; t \in[0, T], T>0 ; u=u(t, x), v=v(t, x)$ are control functions of the 
players; $S_{T}=\{(x, t) \mid x \in \partial \Omega, t \in(0, T)\}, A$ is a differential operator defined in the space $L_{2}(\Omega)$. In this problem, the authors used the fact in [15] that under certain conditions, the problem (1.1) has a unique generalized (in the sense of distribution) solution of the form

$$
z(t, x)=\sum_{k=1}^{\infty} z_{k}(t) \psi_{k}(x)
$$

where the functions $z_{k}(t), 0 \leq t \leq T, k=1,2, \ldots$, constitute the solution of the Cauchy problem for the following infinite system of differential equations and initial conditions:

$$
\dot{z}_{k}+\lambda_{k} z_{k}=-u_{k}(t)+v_{k}(t), \quad z_{k}(0)=z_{0 k}, \quad k=1,2, \ldots,
$$

and $\lambda_{k}, k=1,2, \ldots$, satisfying the condition that $0<\lambda_{1} \leq \lambda_{2} \leq \cdots \rightarrow \infty$, are eigenvalues of the operator $A$, the functions $\psi_{k}(x), k=1,2, \ldots$, constitute an orthonormal and complete system of eigenfunctions of the operator $A, u_{k}(t), v_{k}(t)$, and $z_{0 k}$ are the Fourier coefficients in the expansion of $u(t, x), v(t, x)$, and $z_{0}(x)$, respectively, in the system $\left\{\psi_{k}(x)\right\}$.

The work above shows the significant relationship between differential game problems described by (1.1) in one side and those described by (1.3) in the other side. Therefore, it is logical to study the latter in an independent frame work (see, e.g., $[16,17]$ ).

In the present paper, we solve pursuit and evasion problems described by system (1.3), with function coefficients $\lambda_{k}, k=1,2, \ldots$ instead of constants coefficients considered in the previous study. Different forms of constraints on the controls of the players are considered.

\section{Statement of the Problem}

Let

$$
l_{2}=\left\{\alpha=\left(\alpha_{1}, \alpha_{2}, \ldots\right): \sum_{k=1}^{\infty} \alpha_{k}^{2}<\infty\right\},
$$

with inner product and norm

$$
\begin{gathered}
\langle\alpha, \beta\rangle=\sum_{k=1}^{\infty} \alpha_{k} \beta_{k}, \alpha, \beta \in l_{2}, \quad\|\alpha\|=\left(\sum_{k=1}^{\infty} \alpha_{k}^{2}\right)^{1 / 2}, \\
\|w(\cdot)\|_{L_{2}\left(0, T, l_{2}\right)}=\left(\sum_{k=1}^{\infty} \int_{0}^{T} w_{k}^{2}(s) d s\right)^{1 / 2} .
\end{gathered}
$$

Let

$$
L_{2}\left(0, T, l_{2}\right)=\left\{w(t)=\left(w_{1}(t), w_{2}(t), \ldots\right):\|w(\cdot)\|_{L_{2}\left(0, T, l_{2}\right)}<\infty, w_{k}(\cdot) \in L_{2}(0, T)\right\}
$$

where $T, T>0$, is a given number. 
We examine a pursuit and evasion differential game problems described by the following infinite system of differential equations

$$
\dot{z}_{k}(t)+\lambda_{k}(t) z_{k}(t)=-u_{k}(t)+v_{k}(t), \quad z_{k}(0)=z_{k 0}, \quad k=1,2, \ldots,
$$

where $z_{k}, u_{k}, v_{k} \in R^{1}, k=1,2, \ldots, z_{0}=\left(z_{10}, z_{20}, \ldots\right) \in l_{2}, u_{k}, v_{k}, \ldots$, are control parameters of pursuer and evader respectively, $\lambda_{k}(t), k=1,2, \ldots$, are bounded, non-negative continuous functions on the interval $[0, T]$ such that $\lambda_{k}(0)=0, k=1,2, \ldots$

Definition 2.1. A function $w(\cdot), w:[0, T] \rightarrow l_{2}$, with measurable coordinates $w_{k}(t), 0 \leq t \leq$ $T, k=1,2, \ldots$, subject to

$$
\sum_{k=1}^{\infty} \int_{0}^{T} w_{k}^{2}(s) d s \leq \rho^{2}\left(\sum_{k=1}^{\infty} w_{k}^{2}(t) \leq \rho^{2}, t \in[0, T]\right)
$$

where $\rho$ is a positive number, is referred to as an admissible control subject to integral constraint (resp., geometric constraint).

We denote the set of all admissible controls with respect to integral constraint by $S_{1}(\rho)$ and with respect to geometric constraint by $S_{2}(\rho)$.

The control $u(\cdot)=\left(u_{1}(\cdot), u_{2}(\cdot), \ldots\right)$ of the pursuer and $v(\cdot)=\left(v_{1}(\cdot), v_{2}(\cdot), \ldots\right)$ of the evader are said to be admissible if they satisfy one of the following conditions

$$
\begin{gathered}
\left(\sum_{k=1}^{\infty} \int_{0}^{T} u_{k}^{2}(s) d s\right)^{1 / 2} \leq \rho, \quad\left(\sum_{k=1}^{\infty} \int_{0}^{T} v_{k}^{2}(s) d s\right)^{1 / 2} \leq \sigma, \\
\left(\sum_{k=1}^{\infty} u_{k}^{2}(t)\right)^{1 / 2} \leq \rho, t \in[0, T], \quad\left(\sum_{k=1}^{\infty} v_{k}^{2}(t)\right)^{1 / 2} \leq \sigma, t \in[0, T], \\
\left(\sum_{k=1}^{\infty} \int_{0}^{T} u_{k}^{2}(s) d s\right)^{1 / 2} \leq \rho, \quad\left(\sum_{k=1}^{\infty} v_{k}^{2}(t)\right)^{1 / 2} \leq \sigma, t \in[0, T], \\
\left(\sum_{k=1}^{\infty} u_{k}^{2}(t)\right)^{1 / 2} \leq \rho, t \in[0, T], \quad\left(\sum_{k=1}^{\infty} \int_{0}^{T} v_{k}^{2}(s) d s\right)^{1 / 2} \leq \sigma,
\end{gathered}
$$

where $\rho$ and $\sigma$ are positive constants. We will call the system (2.4) in which $u(\cdot)$ and $v(\cdot)$ satisfy inequalities (2.6) (resp., (2.7), (2.8), and (2.9)), game $G_{1}$ (resp., $G_{2}, G_{3}, G_{4}$ ).

Definition 2.2. A function $z(t)=\left(z_{1}(t), z_{2}(t), \ldots\right), 0 \leq t \leq T$, is called the solution of the system (2.4) if each coordinate $z_{k}(t)$

(i) is absolutely continuous and almost everywhere on $[0, T]$ satisfies (2.4),

(ii) $z(\cdot) \in C\left(0, T ; l_{2}\right)$. 
Definition 2.3. A function

$$
U(t, v), \quad U:[0, T] \times l_{2} \rightarrow l_{2}
$$

is referred to as the strategy of the pursuer with respect to integral constraint if:

(1) for any admissible control of the evader $v=v(t), t \in[0, T]$, the system (2.4) has a unique solution at $u=u\left(t, v_{1}(t), v_{2}(t), \ldots\right)$,

(2) $U(\cdot, v(\cdot)) \in S_{1}(\rho)$.

In a similar way, we define strategy of the pursuer with respect to geometric constraint.

Definition 2.4. One will say that pursuit can be completed in the game $G_{1}$ (resp., $G_{2}, G_{3}$ ) from an initial position $z_{0}$, if there exists a strategy of the pursuer to ensure that $z(t)=0$ for some $t \in[0, T]$ and for any admissible control of the evader $v(\cdot)$, where $z(t)$ is the solution to (2.4).

Definition 2.5. One will say that pursuit can be completed in the game $G_{4}$ from an initial position $z_{0}$, if for arbitrary $\varepsilon>0$, there exists a strategy of the pursuer to ensure that $\|z(t)\| \leq \varepsilon$ for some $t \in[0, T]$ and for any admissible control of the evader $v(\cdot)$, where $z(t)$ is the solution to $(2.4)$.

Definition 2.6. One will say that evasion is possible in the game $G_{1}$ (resp., $G_{2}, G_{3}, G_{4}$ ) from the initial position $z_{0} \neq 0$, if there exists a function $v(t) \in S_{1}(\sigma)\left(v(t) \in S_{2}(\sigma), v(t) \in S_{2}(\sigma), v(t) \in\right.$ $\left.S_{1}(\sigma)\right)$ such that, for arbitrary function $u_{0}(t) \in S_{1}(\rho)\left(u_{0}(t) \in S_{2}(\rho), u_{0}(t) \in S_{1}(\rho), u_{0}(t) \in\right.$ $\left.S_{2}(\rho)\right)$, the solution $z(t)$ of $(2.4)$ does not vanish, that is, $z(t) \neq 0$ for any $t \in[0, T]$.

The problem is to find

(1) conditions on the initial state $z_{0}$ for which pursuit can be completed for a finite time;

(2) conditions for which evasion is possible from any initial position $z_{0} \neq 0$ in the differential game $G_{i}$, for $i=1,2,3,4$.

In problems 1 and 2, different forms of constraints on the controls of the players are to be considered.

\section{Differential Game Problem}

The $k$ th equation in (2.4) has a unique solution of the form

$$
z_{k}(t)=e^{-\alpha_{k}(t)}\left(z_{k 0}-\int_{0}^{t} u_{k}(s) e^{\alpha_{k}(s)} d s+\int_{0}^{t} v_{k}(s) e^{\alpha_{k}(s)} d s\right)
$$

where $\alpha_{k}(t)=\int_{0}^{t} \lambda_{k}(s) d s$.

It has been proven in [18] that the solution $z(t)=\left(z_{1}(t), z_{2}(t), \ldots\right)$ of $(2.4)$, where $z_{k}, k=1,2, \ldots$ defined by (3.1), belongs to the space $C\left(0, T ; l_{2}\right)$. 
Journal of Applied Mathematics

Let

$$
\begin{aligned}
Y & =\left\{z_{0}=\left(z_{10}, z_{20}, \ldots\right) \mid \exists k=j: z_{j 0}^{2} \leq \frac{\rho^{2}}{4}, A_{j}(t) \geq 1\right\}, \\
Y_{1}(T) & =\left\{z_{0}=\left(z_{10}, z_{20}, \ldots\right) \mid \sum_{k=1}^{\infty} \frac{z_{k 0}^{2}}{A_{k}(T)} \leq(\rho-\sigma)^{2}\right\}, \\
Y_{2}(T) & =\left\{z_{0}=\left(z_{10}, z_{20}, \ldots\right) \mid \sum_{k=1}^{\infty} \frac{z_{k 0}^{2}}{B_{k}^{2}(T)} \leq(\rho-\sigma)^{2}\right\}, \\
Y_{3}(T) & =\left\{z_{0}=\left(z_{10}, z_{20}, \ldots\right) \mid\left\|z_{0}\right\|+\frac{\sigma^{2}}{\varepsilon} \sup _{k} A_{k}(T) \leq \rho T, \varepsilon>0\right\},
\end{aligned}
$$

where $A_{k}(T)=\int_{0}^{T} e^{2 \alpha_{k}(s)} d s$ and $B_{k}(T)=\int_{0}^{T} e^{\alpha_{k}(s)} d s$.

\subsection{Pursuit Differential Game}

Theorem 3.1. If $\rho \geq \sigma$ then from the initial position $z_{0} \in Y_{1}(T)$, pursuit can be completed in the game $G_{1}$.

Proof. Let define the pursuer's strategy as

$$
u_{k}(t)= \begin{cases}z_{k 0} \mathrm{~A}_{k}^{-1}(T) e^{\alpha_{k}(t)}+v_{k}(t), & 0 \leq t \leq T, \\ 0, & t>T .\end{cases}
$$

The admissibility of this strategy follows from the relations

$$
\begin{aligned}
\left(\sum_{k=1}^{\infty} \int_{0}^{T} u_{k}^{2}(s) d s\right)^{1 / 2} & =\left(\sum_{k=1}^{\infty} \int_{0}^{T}\left|z_{k 0} A_{k}^{-1}(T) e^{\alpha_{k}(s)}+v_{k}(s)\right|^{2} d s\right)^{1 / 2} \\
& \leq\left(\sum_{k=1}^{\infty} \int_{0}^{T}\left(\left|z_{k 0} A_{k}^{-1}(T) e^{\alpha_{k}(s)}\right|+\left|v_{k}(s)\right|\right)^{2} d s\right)^{1 / 2} \\
& \leq\left(\sum_{k=1}^{\infty} \int_{0}^{T}\left|z_{k 0} A_{k}^{-1}(T) e^{\alpha_{k}(s)}\right|^{2} d s\right)^{1 / 2}+\left(\sum_{k=1}^{\infty} \int_{0}^{T}\left|v_{k}(s)\right|^{2} d s\right)^{1 / 2} \\
& \leq\left(\sum_{k=1}^{\infty} z_{k 0}^{2} A_{k}^{-1}(T)\right)^{1 / 2}+\sigma \\
& =\rho-\sigma+\sigma=\rho,
\end{aligned}
$$

here we used the Minkowski inequality and the fact that $z_{0} \in Y_{1}(T)$. 
Suppose that the pursuer uses the strategy (3.3), one can easily see that for any admissible control of the evader $z_{k}(T)=0, k=1,2, \ldots$, that is,

$$
\begin{aligned}
z_{k}(T) & =e^{-\alpha_{k}(T)}\left(z_{k 0}-\int_{0}^{T} z_{k 0} A_{k}^{-1}(T) e^{2 \alpha_{k}(s)} d s\right) \\
& =e^{-\alpha_{k}(T)}\left(z_{k 0}-z_{k 0}\right)=0 .
\end{aligned}
$$

Therefore, pursuit can be completed in the game $G_{1}$. This ends the proof of the theorem.

Theorem 3.2. If $\rho \geq \sigma$ then from the initial position $z_{0} \in Y_{2}(T)$, pursuit can be completed in the game $G_{2}$.

Proof. We define the pursuer's strategy as

$$
u_{k}(t)= \begin{cases}z_{k 0} B_{k}^{-1}(T)+v_{k}(t), & 0 \leq t \leq T, \\ 0, & t>T .\end{cases}
$$

The inclusion $u(\cdot) \in S_{2}(\rho)$ follows from the relations

$$
\begin{aligned}
\left(\sum_{k=1}^{\infty} u_{k}^{2}(t)\right)^{1 / 2} & =\left(\sum_{k=1}^{\infty}\left|z_{k 0} B_{k}^{-1}(T)+v_{k}(t)\right|^{2}\right)^{1 / 2} \\
& \leq\left(\sum_{k=1}^{\infty}\left(\left|z_{k 0} B_{k}^{-1}(T)\right|+\left|v_{k}(t)\right|\right)^{2}\right)^{1 / 2} \\
& \leq\left(\sum_{k=1}^{\infty}\left|z_{k 0} B_{k}^{-1}(T)\right|^{2}\right)^{1 / 2}+\left(\sum_{k=1}^{\infty}\left|v_{k}(t)\right|^{2}\right)^{1 / 2} \\
& =\rho-\sigma+\sigma=\rho,
\end{aligned}
$$

here we used the Minkowski inequality and the fact that $z_{0} \in Y_{2}(T)$.

Suppose that the pursuer uses the strategy (3.6). One can easily see that $z_{k}(T)=0, k=$ $1,2, \ldots$, that is,

$$
\begin{aligned}
z_{k}(T) & =e^{-\alpha_{k}(T)}\left(z_{k 0}-\int_{0}^{T} z_{k 0} B_{k}^{-1}(T) e^{\alpha_{k}(s)} d s\right) \\
& =e^{-\alpha_{k}(T)}\left(z_{k 0}-z_{k 0}\right)=0 .
\end{aligned}
$$

Therefore, pursuit can be completed in the game $G_{2}$. This completes the proof of the theorem.

Theorem 3.3. If $\rho \geq \sigma$ and $z_{0} \in Y_{1}(T)$ at some $T \in(0, T]$, then pursuit can be completed in the game $G_{3}$. 
Proof. Suppose, as contained in the hypothesis of the theorem, that $z_{0} \in Y_{1}(T), T \in(0,1]$ and let $v_{0}(t)$ be an arbitrary admissible control of the evader.

Let the pursuer use the strategy $u(t)=\left(u_{1}(t), u_{2}(t), \ldots\right)$ defined by

$$
u_{k}(t)= \begin{cases}z_{k 0} A_{k}^{-1}(T) e^{\alpha_{k}(t)}+v_{0 k}(t), & 0 \leq t \leq T, \\ 0, & t>T .\end{cases}
$$

Then, using (3.1), we have

$$
\begin{aligned}
z(T) & =e^{-\alpha_{k}(T)}\left(z_{k 0}-\int_{0}^{T} z_{k 0} A_{k}^{-1}(T) e^{2 \alpha_{k}(t)} d s\right) \\
& =e^{-\alpha_{k}(T)}\left(z_{k 0}-z_{k 0}\right)=0 .
\end{aligned}
$$

We now show the admissibility of the strategy used by the pursuer. From the inclusion $v_{0}(t) \in$ $S_{2}(\sigma)$ we can deduce that

$$
\begin{aligned}
\left(\sum_{k=1}^{\infty} \int_{0}^{T} v_{0 k}^{2}(s) d s\right)^{1 / 2} \leq \sigma \sqrt{T} \\
\left(\sum_{0}^{\infty} \int_{k}^{T} u_{k}^{2}(s) d s\right)^{1 / 2}=\left(\sum_{k=1}^{\infty} \int_{0}^{T}\left|z_{k 0} A_{k}^{-1}(T) e^{\alpha_{k}(s)}+v_{0 k}(s)\right|^{2} d s\right)^{1 / 2} \\
\leq\left(\sum_{k=1}^{\infty} \int_{0}^{T}\left(\left|z_{k 0} A_{k}^{-1}(T) e^{\alpha_{k}(s)}\right|+\left|v_{k}(s)\right|\right)^{2} d s\right)^{1 / 2} \\
\leq\left(\sum_{k=1}^{\infty} \int_{0}^{T}\left|z_{k 0} A_{k}^{-1}(T) e^{\alpha_{k}(s)}\right|^{2} d s\right)^{1 / 2}+\left(\sum_{k=1}^{\infty} \int_{0}^{T}\left|v_{0 k}(s)\right|^{2} d s\right)^{1 / 2} \\
\leq\left(\sum_{k=1}^{\infty} z_{k 0}^{2} A_{k}^{-1}(T)\right)^{1 / 2}+\sigma \sqrt{T} \\
=\rho-\sigma+\sigma \sqrt{T} \leq \rho,
\end{aligned}
$$

recall that $T \in(0,1]$ and (3.11). This completes the proof.

Theorem 3.4. For arbitrary $\rho>0, \sigma>0$ and initial position $z_{0} \in Y_{3}(T)$, pursuit can be completed in the game $G_{4}$.

Proof. Let $v_{0}$ be an arbitrary admissible control function of the evader. When the pursuer uses the admissible control function

$$
u_{k}(t)=z_{k 0} T_{1}^{-1} e^{-\alpha_{k}(t)}, \quad k=1,2, \ldots, 0 \leq t \leq T_{1}
$$


for time $T_{1}=\left\|z_{0}\right\| \rho^{-1}$, the solution (3.1) of (2.4) becomes

$$
z_{k}\left(T_{1}\right)=e^{-\alpha_{k}\left(T_{1}\right)} \int_{0}^{T_{1}} v_{0 k}(s) e^{\alpha_{k}(s)} d s
$$

Then for arbitrary positive number $\varepsilon$, it is obvious that either

(1) $\left\|z\left(T_{1}\right)\right\| \leq \varepsilon$, or

(2) $\left\|z\left(T_{1}\right)\right\|>\varepsilon$.

If (1) is true then the proof is complete. Obviously $T_{1} \leq T$.

Suppose that (1) is not true then (2) must hold. We now assume that $z_{0}=z\left(T_{1}\right)$ and repeat previous argument by setting

$$
u_{k}(t)=z_{k 0}\left(T_{1}\right) T_{2}^{-1} e^{-\alpha_{k}(t)}, \quad k=1,2, \ldots, 0 \leq t \leq T_{2},
$$

with time $T_{2}=\left\|z\left(T_{1}\right)\right\| \rho^{-1}$ (we will later prove that the sum of $T_{i}$ is less than or equal to $T$ ). For this step the solution (3.1) becomes

$$
z_{k}\left(T_{1}+T_{2}\right)=e^{-\alpha_{k}\left(T_{2}\right)} \int_{0}^{T_{2}} v_{0 k}\left(T_{1}+s\right) e^{\alpha_{k}(s)} d s
$$

Yet again, we have either of the following cases holding:

(1) $\left\|z\left(T_{1}+T_{2}\right)\right\| \leq \varepsilon$, or

(2) $\left\|z\left(T_{1}+T_{2}\right)\right\|>\varepsilon$.

If (1) holds then the game is completed in the time $T_{1}+T_{2}$, else we assume $z_{0}=z_{0}\left(T_{1}+T_{2}\right)$ and repeat the process again and so on.

We now proof a claim that the game will be completed before $n$th finite step, where

$$
n=\left\lceil\frac{\sigma^{2} \sup _{k} A_{k}(T)}{\varepsilon^{2}}\right\rceil \text {. }
$$

Note that the existence of the supreme of the sequence $A_{1}(T), A_{2}(T), \ldots$, follows from the fact that $\lambda_{1}(t), \lambda_{2}(t), \ldots$ is a bounded sequence of continuous functions and $t \in[0, T]$. must have

Suppose that it is possible that the game can continue for $n$th step. In this case, we

$$
\sum_{i=1}^{n} \sigma_{i}^{2} \leq \sigma^{2}
$$

But in the first instance, we have

$$
\left|z_{k}\left(T_{1}\right)\right|^{2} \leq e^{-2 \alpha_{k}\left(T_{1}\right)} \int_{0}^{T_{1}} v_{0 k}^{2}(s) d s \int_{0}^{T_{1}} e^{2 \alpha_{k}(s)} d s \leq \sup _{k} A_{k}(T) \int_{0}^{T_{1}} v_{0 k}^{2}(s) d s,
$$

here we used (3.14) and Cauchy-Schwarz inequality. 
Therefore,

$$
\left\|z\left(T_{1}\right)\right\|^{2} \leq \sup _{k} A_{k}(T) \sum_{k=1}^{\infty} \int_{0}^{T_{1}} v_{0 k}^{2}(s) d s=\sup _{k} A_{k}(T) \sigma_{1}^{2},
$$

and by using the assumption that $\left\|z\left(T_{1}\right)\right\|>\varepsilon$, we have

$$
\sigma_{1}^{2}>\frac{\varepsilon^{2}}{\sup _{k} A_{k}(T)}
$$

Since the right hand side of this inequality is independent of $n$, we can conclude that

$$
\sigma_{n}^{2}>\frac{\varepsilon^{2}}{\sup _{k} A_{k}(T)}
$$

Using this inequality and definition of $n$, we have

$$
\sum_{i=1}^{n} \sigma_{i}^{2}>\frac{n \varepsilon^{2}}{\sup _{k} A_{k}(T)}>\sigma^{2}
$$

contradicting (3.18). Hence, pursuit must be completed for the initial position $z_{0} \in Y_{3}(T)$ before the $n$th step. Furthermore, the pursuit time is given by $T\left(z_{0}\right)=T_{1}+T_{2}+\cdots+T_{n-1}$, and the inclusion $T\left(z_{0}\right) \in[0, T]$ is satisfied. Indeed (see (3.20), definition of $n$ and that $z_{0} \in Y_{3}(T)$ ),

$$
\begin{aligned}
T\left(z_{0}\right) & =\frac{\left\|z_{0}\right\|}{\rho}+\frac{\left\|z\left(T_{1}\right)\right\|}{\rho}+\cdots+\frac{\left\|z\left(T_{n-2}\right)\right\|}{\rho} \\
& \leq \frac{1}{\rho}\left(\left\|z_{0}\right\|+\sqrt{\sup _{k} A_{k}(T)} \sum_{i=1}^{n-2} \sigma_{i}\right) \\
& \leq \frac{1}{\rho}\left(\left\|z_{0}\right\|+\sigma \sqrt{(n-2) \sup _{k} A_{k}(T)}\right) \\
& \leq \frac{1}{\rho}\left(\left\|z_{0}\right\|+\frac{\sigma^{2}}{\varepsilon} \sup _{k} A_{k}(T)\right) \leq T .
\end{aligned}
$$

This proves the theorem.

\subsection{Evasion Differential Game}

Theorem 3.5. If $\sigma-\rho \geq 0$ then evasion is possible in the game $G_{1}$ from the initial position $z_{0} \neq 0$.

Proof. Suppose that

$$
\sigma-\rho \geq 0
$$


and let $u_{0}(t)$ be an arbitrary control of the pursuer subjected to integral constraint. We construct the control function of the evader as follows:

$$
v_{k}(t)= \begin{cases}A_{j}^{-1 / 2}(T) \rho e^{\alpha_{j}(t)}, & k=j, \\ 0, & k \neq j .\end{cases}
$$

This control function belongs to $S_{1}(\sigma)$. Indeed,

$$
\sum_{k=1}^{\infty} \int_{0}^{T} v_{k}^{2}(s) d s=A_{j}^{-1}(T) \rho^{2} A_{j}(T) \leq \sigma^{2}
$$

we have used (3.26) and (3.25).

Our goal now is to show that $z_{j}(t) \neq 0$ for any $t \in[0, T]$ as defined by (3.1). Substituting (3.26) into (3.1) and using the Cauchy-Schwartz inequality, we have

$$
\begin{aligned}
z_{j}(t) & \geq e^{-\alpha_{j}(t)}\left(z_{j 0}+\rho \sqrt{A_{j}(t)}-\rho \sqrt{A_{j}(t)}\right) \\
& =z_{j 0} e^{-\alpha_{j}(t)}>0
\end{aligned}
$$

for any $t \in[0, T]$. It follows that $z(t) \neq 0$ on the interval $[0, T]$. Hence, evasion is possible in the game $G_{1}$ from the given initial position $z_{0} \neq 0$. The proof of the theorem is complete.

Theorem 3.6. Suppose that $\sigma \geq \rho$ or there exists a number $k=j$ such that $z_{0 j}>0$ and $\sigma-$ $\rho \sqrt{A_{j}(T)} \geq 0$. Then from the initial position $z_{0} \neq 0$, evasion is possible in the game $G_{2}$.

Proof. Suppose that $\sigma \geq \rho$ and that $z_{0} \neq 0$. The later condition means that $z_{k 0} \neq 0$ for some $k=j$. We construct the control function of the evader as follows:

$$
v_{k}(t)= \begin{cases}\rho, & k=j, \\ 0, & k \neq j .\end{cases}
$$

It is obvious that this control belongs to the set $S_{2}(\sigma)$.

To be definite, let $z_{j 0}>0$. Using (3.29) and (3.1), we have

$$
\begin{aligned}
z_{j}(t) & >e^{-\alpha_{j}(t)}\left(z_{j 0}+\rho \int_{0}^{t} e^{\alpha_{j}(s)} d s-\rho \int_{0}^{t} e^{\alpha_{j}(s)} d s\right) \\
& =e^{-\alpha_{j}(t)} z_{j 0}>0 .
\end{aligned}
$$

This means that evasion is possible from the initial position $z_{0} \neq 0$ in the game $G_{2}$. 
We now prove the theorem with the alternative condition. Suppose that there exists a number $k=j$ such that $z_{0 j}>0$ and $\sigma-\rho \sqrt{A_{j}(T)} \geq 0$. Let the control of the evader be as follows:

$$
v_{k}(t)= \begin{cases}\frac{\sqrt{T A_{j}(T)}}{t+e+T} \rho e^{-\alpha_{j}(t)}, & k=j, \\ 0, & k \neq j .\end{cases}
$$

We show that this control satisfies the geometric constraint:

$$
\sum_{k=1}^{\infty} v_{k}^{2}(t)=\frac{T A_{j}(T)}{(t+e+T)^{2}} \rho^{2} e^{-2 \alpha_{j}(t)} \leq \rho^{2} A_{j}(T) \leq \sigma^{2}
$$

When the evader uses the control (3.31), the non-vanishing of $z_{j}(t)$ in the interval $[0, T]$ for any admissible control of the pursuer $u_{i 0}$, can be seen from the following (see (3.1))

$$
z_{j}(t) \geq e^{-\alpha_{j}(t)}\left(z_{j 0}+\rho \sqrt{T A_{j}(T)} \ln (t+e+T)-\rho \sqrt{T A_{j}(T)}\right)>0,
$$

we use the fact that $\ln (t+e+T)>1$ for any $t \in[0, T]$.

Therefore, $z(t) \neq 0, t \in[0, T]$. This completes the proof of the theorem.

Theorem 3.7. If $\sigma-\rho \sqrt{T} \geq 0$ then evasion is possible from the initial position $z_{0} \neq 0$ in the game $G_{4}$.

Proof. Suppose that $z_{0} \neq 0$ and that $\sigma-\rho \sqrt{T} \geq 0$. We construct the control function of the evader as follows:

$$
v_{k}(t)= \begin{cases}\rho \sqrt{\frac{T}{A_{j}(T)}} e^{\alpha_{j}(t)}, & k=j, \\ 0, & k \neq j .\end{cases}
$$

We now show that this control satisfies the integral constraint

$$
\sum_{k=1}^{\infty} \int_{0}^{T} v_{k}^{2}(s) d s=\rho^{2} \frac{T}{A_{j}(T)} \int_{0}^{T} e^{2 \alpha_{j}(s)} d s \leq \rho^{2} T \leq \sigma^{2} .
$$

When the evader uses the control (3.34), our task is to show that $z_{j}(t)$ does not vanish in the interval $[0, T]$ for any admissible control of the pursuer $u_{i 0}$.

For definiteness let $z_{j 0}>0$. Substituting (3.34) into (3.1), we have

$$
z_{j}(t) \geq e^{-\alpha_{j}(t)}\left(z_{j 0}+\rho \sqrt{\frac{T}{A_{j}(T)}} A_{j}(T)-\rho \sqrt{T A_{j}(T)}\right)=e^{-\alpha_{j}(t)} z_{j 0}>0 .
$$

Therefore, $z(t) \neq 0, t \in[0, T]$. This means that evasion is possible from initial position $z_{0} \neq 0$ in game $G_{4}$. This ends the proof of the theorem. 
Theorem 3.8. If $z_{0} \in Y$ and $\sigma \geq 2 \rho e^{\alpha_{j}(T)}$ for some $k=j$, then from the initial position $z_{0} \neq 0$ evasion is possible in the game $G_{3}$.

Proof. Suppose that $z_{0} \in Y$ and that there exists $k=j$ such that

$$
\sigma \geq 2 \rho e^{\alpha_{j}(T)}
$$

We construct the control function of the evader as follows:

$$
v_{k}(t)= \begin{cases}\left(2 z_{j 0}+\rho\right) e^{\alpha_{j}(t)}, & k=j, \\ 0, & k \neq j .\end{cases}
$$

The inclusion $v(\cdot) \in S_{2}(\sigma)$ follows from the following

$$
\begin{aligned}
\sum_{k=1}^{\infty} v_{k}^{2}(t) & =\left[\left(2 z_{j 0}+\rho\right) e^{\alpha_{j}(t)}\right]^{2} \\
& \leq 8 z_{j 0}^{2} e^{2 \alpha_{j}(t)}+2 \rho^{2} e^{2 \alpha_{j}(t)} \\
& \leq 2 \rho^{2} e^{2 \alpha_{j}(t)}+2 \rho^{2} e^{2 \alpha_{j}(t)} \\
& \leq 4 \rho^{2} e^{2 \alpha_{j}(T)} \leq \sigma^{2}
\end{aligned}
$$

we used (3.34); (3.37) and the inequality $(a+b)^{2} \leq 2 a^{2}+2 b^{2}$.

Let the evader use the control (3.34) and for definiteness let $z_{j 0}>0$. Using (3.1) and the Cauchy-Schwartz inequality, we have

$$
\begin{aligned}
z_{j}(t) & \geq e^{-\alpha_{j}(t)}\left(z_{j 0}-\rho \sqrt{A_{j}(t)}+2 z_{j 0} A_{j}(t)+\rho \sqrt{A_{j}(t)}\right) \\
& =e^{-\alpha_{j}(t)}\left(z_{j 0}+2 z_{j 0} \mathrm{~A}_{j}(t)\right)>0 .
\end{aligned}
$$

Therefore, we have $z_{j}(t)>0,0 \leq t \leq T$, that is, evasion is possible in the game $G_{3}$. This ends the proof of the theorem.

\section{Conclusion}

This paper is closely related to [10,11]. However, the game model considered in this paper is a better generalization to the one in the last cited papers. The constant coefficients of the game model considered in the cited papers are specific to function coefficients considered in this papers. Sufficient conditions for which pursuit can be completed and for which evasion is possible with various form of constraints on the control of the players have been established.

For future works, optimal pursuit and multiplayers game problems described by the model considered in this paper can be investigated. As there are four different possible combinations of geometric and integral constraints on the control functions of the two players of the game, there would be four different problems to be studied. 


\section{Acknowledgment}

The authors wish to express the deepest appreciation to the reviewers for their valuable comments and observations. This research was partially supported by the Research Grant (RUGS) of the Universiti Putra Malaysia, no. 05-04-10-1005RU.

\section{References}

[1] A. Friedman, Differential Games, Wiley-Interscience, New York, NY, USA, 1971.

[2] R. Isaacs, Differential Games, John Wiley \& Sons, New York, NY, USA, 1965.

[3] N. N. Krasovskir and A. I. Subbotin, Game-Theoretical Control Problems, Springer, New York, NY, USA, 1988.

[4] J. Lewin, Differential Games, Springer, London, UK, 1994.

[5] L. A. Petrosyan, Differential Games of Pursuit, Petersburg State University press, 1993.

[6] L. S. Pontryagin, Collected Works, Nauka, 1988.

[7] G. I. Ibragimov, "A problem of optimal pursuit in systems with distributed parameters," Journal of Applied Mathematics and Mechanics, vol. 66, no. 5, pp. 719-724, 2002.

[8] G. I. Ibragimov, "Damping of an oscillation system in presence of disturbance," Uzbek Mathematical Journal, no. 1, pp. 34-45, 2005.

[9] Y. S. Osipov, "The theory of differential games in distributed-parameter systems," Doklady Akademii Nauk SSSR, vol. 223, no. 6, pp. 1314-1317, 1975.

[10] N. Y. Satimov and M. Tukhtasinov, “On some game problems for first-order controlled evolution equations," Differentsial'nye Uravneniya, vol. 41, no. 8, pp. 1114-1121, 2005.

[11] N. Y. Satimov and M. Tukhtasinov, "Game problems on a fixed interval in controlled first-order evolution equations," Mathematical Notes, vol. 80, no. 4, pp. 578-589, 2006.

[12] N. Y. Satimov and M. Tukhtasinov, "On game problems for second-order evolution equations," Russian Mathematics, vol. 51, no. 1, pp. 49-57, 2007.

[13] M. Tukhtasinov, "On some problems in the theory of differential pursuit games in systems with distributed parameters," Journal of Applied Mathematics and Mechanics, vol. 59, no. 6, pp. 979-984, 1995.

[14] M. Tukhtasinov and M. S. Mamatov, "On pursuit problems in controlled distributed parameters systems," Mathematical Notes, vol. 84, no. 2, pp. 256-262, 2008.

[15] O. A. Ladyzhenskaya, Boundary-Value Problems of Matahematical Physics, Nauka, Moscow, Russia, 1973.

[16] G. I. Ibragimov and M. H. Risman, "A differential game of evasion from many pursuers," Malaysian Journal of Mathematical Sciences, vol. 2, no. 3, pp. 49-58, 2008.

[17] G. I. Ibragimov and M. H. Risman, "Pursuit and evasion differential games in Hilbert space," International Game Theory Review, vol. 12, no. 3, pp. 239-251, 2010.

[18] G. I. Ibragimov and A. B. Ja'afaru, "On existence-uniqueness of solution to countable number of firstorder differential equations in the space $l_{2}, "$ Journal of Applied Sciences Research, vol. 7, no. 12, pp. 1860-1864, 2011. 


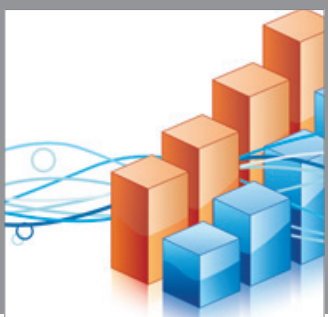

Advances in

Operations Research

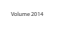

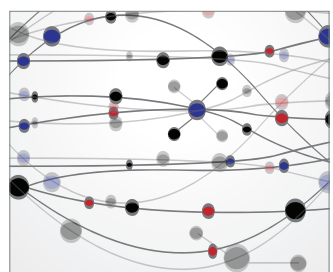

\section{The Scientific} World Journal
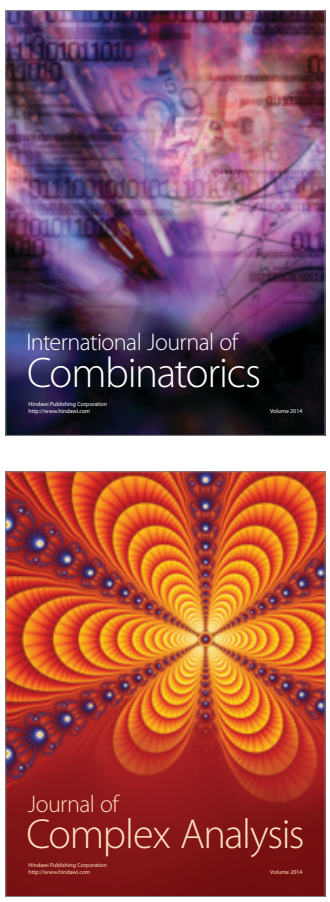

International Journal of

Mathematics and

Mathematical

Sciences
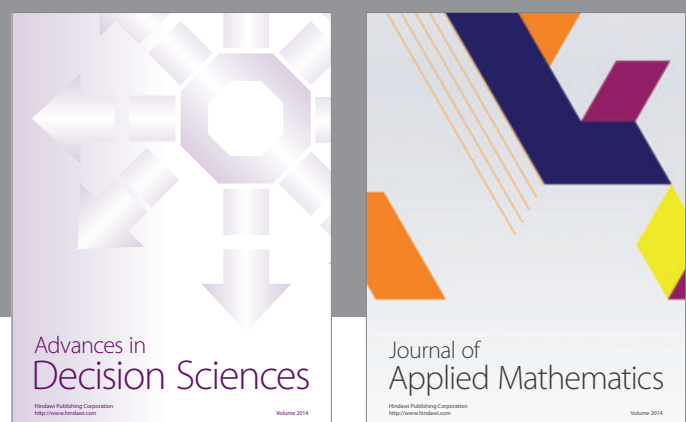

Journal of

Applied Mathematics
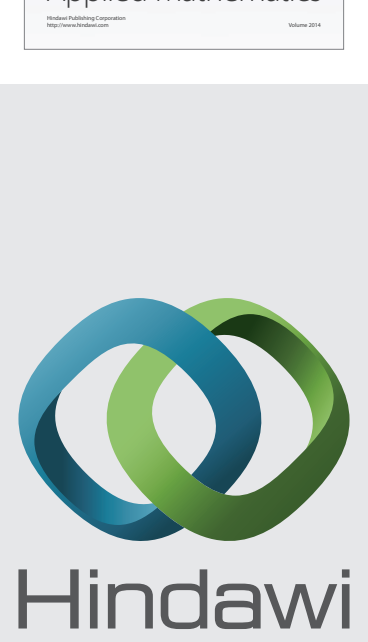

Submit your manuscripts at http://www.hindawi.com
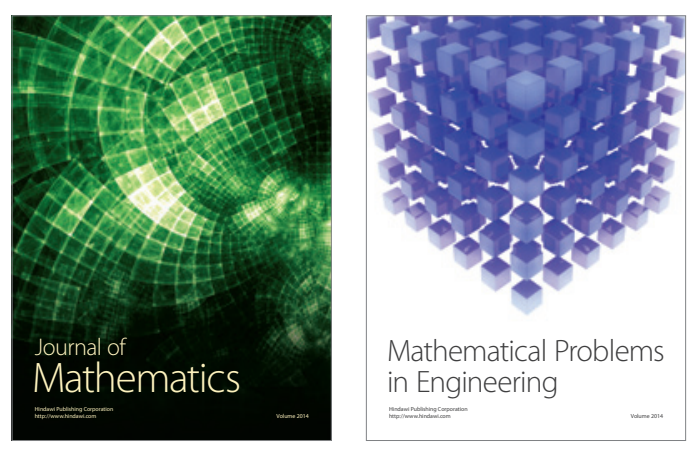

Mathematical Problems in Engineering
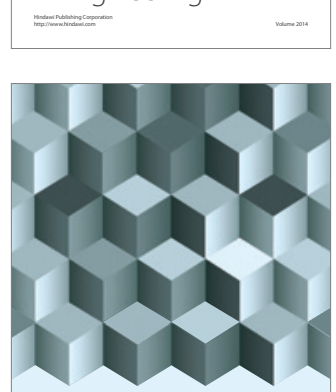

Journal of

Function Spaces
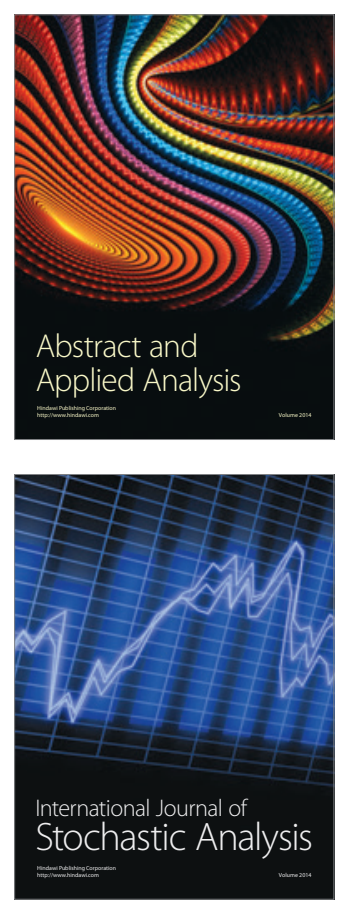

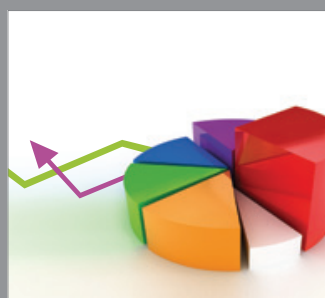

ournal of

Probability and Statistics

Promensencen
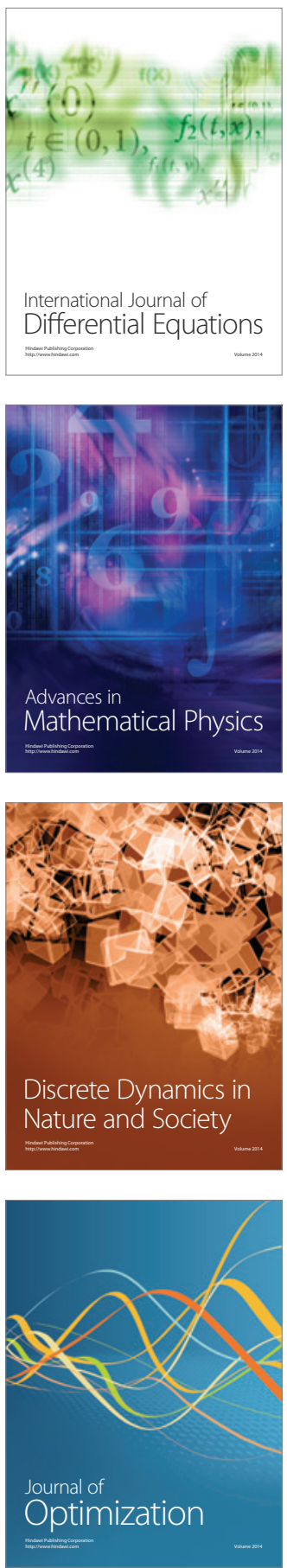